\title{
Implantando CMM Nível 2: A Estratégia SERPRO
}

\author{
Helena Cristina Tavares, Fábio Rilston Silva Paim, Ana Elizabete Carvalho \\ Serviço Federal de Processamento de Dados - Regional Recife (Brazil) \\ helena-cristina.tavares@serpro.gov.br \\ fabio-rilston.paim@serpro.gov.br \\ ana-elizabete.carvalho@serpro.gov.br
}

\begin{abstract}
Resumo
Com a explosão de novas tecnologias e o aumento crescente dos critérios de qualidade dos seus clientes, a indústria de software passou a requerer mais que um corpo técnico capacitado e ferramentas de última geração. As empresas do setor passaram a investir esforços na melhoria do seu processo de desenvolvimento, visando a adesão a modelos como o SEI-CMM, para permitir o uso sistemático das melhores práticas na consecução de projetos de qualidade. Esse trabalho descreve a estratégia do SERPRO para atingir o nível 2 do CMM, os desafios impostos pelas características internas da organização, as soluções encontradas e as lições aprendidas durante este processo.
\end{abstract}

Palavras-chave: qualidade do processo, CMM, processo de software, implantação de programas de qualidade.

\begin{abstract}
A boost in the software technology field and increasingly high quality standards imposed by its clients have required more than highly qualified teams and last generation tools from the software industry to generate quality products. Software companies have applied efforts towards improving their software process, aiming to adhere to quality models such as SEI-CMM, in order to systematically use the best technical and managerial practices to achieve quality within their projects. This work presents SERPRO's strategy to adhere to CMM-Level 2's practices, the challenges introduced by its organizational complexity, the solutions yielded and lessons learned during the process.
\end{abstract}

Key words: process quality, CMM, software process improvement.

\section{Introdução}

Com os recentes avanços na indústria de software, os engenheiros de sistema têm à disposição um arsenal de técnicas, métodos e ferramentas para o desenvolvimento de aplicações. Esses recursos permitem agregar uma infinidade de funcionalidades ao produto de software para atender às necessidades do cliente. Cientes deste fato, esses mesmos clientes estabelecem critérios cada vez mais refinados de qualidade para o produto final. Num mercado competitivo como o atual, as empresas de software percebem que apenas a disponibilidade de recursos técnicos e humanos não é suficiente para atingir as metas de qualidade na produção. É o caso do SERPRO, uma empresa do governo federal que presta serviços de informática a uma vasta gama de clientes da administração direta e indireta.

Num projeto em conjunto com empresas de consultoria, o SERPRO analisou o estágio de evolução de seu processo de software e verificou a necessidade de adoção de um processo sustentado que estabelecesse uma ponte entre o uso sistemático de recursos e a incorporação de práticas gerenciais aos projetos, com o intuito de assegurar o aumento da produtividade, o 
cumprimento de prazos e a conformidade com os altos padrões de qualidade de seus clientes. Inspirado nessas diretrizes, e apoiado por um extenso projeto de modernização do seu parque de informática, o SERPRO desenvolveu o Processo SERPRO de Desenvolvimento de Soluções (PSDS) ([1]), o qual integra atividades consagradas de desenvolvimento de software às práticas de melhoria do processo definidas no modelo CMM (Capability Maturity Model) ([2]). O objetivo maior do PSDS é organizar e promover a melhoria contínua do processo de desenvolvimento, levando-o de um patamar informal (ad hoc) aos níveis maiores de maturidade preconizados pelo CMM.

Devido à complexidade do ambiente de desenvolvimento do SERPRO, a implantação do PSDS foi subdividida em etapas. Num primeiro momento (estágio atual), a estratégia estabelecida foi a implantação de todas as práticas de gerência preconizadas no nível 2 de maturidade do CMM, com vistas a garantir a adesão do SERPRO a esse nível. Esse trabalho descreve a estratégia estabelecida para alcançar este objetivo, os desafios impostos pelas características internas da organização, as soluções encontradas e as lições aprendidas durante este processo.

O trabalho está organizado da seguinte forma: na seção 2 descrevemos brevemente a empresa SERPRO. Na seção 3 apresentamos o PSDS e sua integração com o modelo CMM. A estratégia SERPRO para implantação do nível 2 do CMM é descrita na seção 4, seguida da análise das dificuldades e soluções encontradas durante a implantação de suas área-chave na seção 5. A seção 6 enumera as lições aprendidas. A seção 7 resume nossas conclusões.

\section{A Empresa SERPRO}

O SERPRO, Serviço Federal de Processamento de Dados, é uma empresa de informática vinculada ao Ministério da Fazenda, cuja função principal é a execução de serviços de tratamento de informações e processamento de dados para o governo federal. A Empresa está sediada em Brasília, com representações regionais em 10 capitais (Belém, Belo Horizonte, Curitiba, Fortaleza, Porto Alegre, Recife, Rio de Janeiro, Salvador, São Paulo e a própria capital federal) e um quadro de 8.774 funcionários. Como empresa de informática, a visão do SERPRO é prover "o melhor em tecnologia da informação para o sucesso dos clientes". Essa visão se coaduna com a missão maior da empresa de "fornecer soluções baseadas em tecnologia da informação para êxito das decisões e operações de seus clientes, com inovação, qualidade e segurança, a preços competitivos".

A Empresa encontra-se estruturada em Unidades de Gestão (UG) responsáveis por um segmento da Administração Pública. Cada segmento atende a pelo menos um órgão federal (com características e necessidades próprias), por intermédio de projeções da UG em cada uma das unidades organizacionais. A natureza diversa desses clientes e a descentralização do desenvolvimento em suas diversas representações exigem do SERPRO a manutenção de um complexo parque de desenvolvimento e o envolvimento direto com as mais diferentes plataformas tecnológicas. Neste cenário, a adoção de práticas para padronização, organização e controle do processo de software torna-se fundamental para a perfeita integração entre as unidades de negócio.

\section{O Caminho para a Qualidade}

Nos últimos anos, a explosão de novas tecnologias e o aumento no volume de demandas dos seus clientes tem representado um peso considerável na gestão do desenvolvimento do SERPRO. A geração de produtos e prestação de serviços dentro dos elevados requisitos de qualidade estabelecidos tornou a administração deste processo uma tarefa ao mesmo tempo 
complexa e sujeita a erros. Manter-se na excelência da tecnologia da informação para o serviço público passou a requerer mais que um corpo técnico capacitado e ferramentas de última geração, uma preocupação muito freqüente no início da década de 90 . Tal direcionamento deu lugar à necessidade de um processo organizado de desenvolvimento de software, de forma a permitir o uso sistemático das melhores práticas para a consecução de projetos de qualidade.

Seguindo uma tendência mundial, o SERPRO passou a investir na melhoria do processo de software para aumentar a qualidade, a produtividade e a previsibilidade dos resultados finais. Um Projeto de Modernização do Desenvolvimento, intitulado PMoD, foi então criado com a meta de conduzir a empresa a adotar o modelo SEI-CMM ${ }^{1}$. O CMM é um dos modelos mais aceitos para a melhoria da qualidade e do processo de software em todo o mundo [3], e define os princípios e práticas que devem ser aplicados a uma organização para atingir estágios evolutivos de maturidade em seu processo de software. O CMM está organizado em 5 níveis de maturidade, os quais são definidos em termos de 18 áreas-chave de processo (KPAs - Key Process Areas) que caracterizam a performance do projeto a cada nível $[1,4]$. O objetivo do PMoD é levar o SERPRO a aderir ao nível 2 (repetível) do CMM, onde práticas de gerência mínimas estão estabelecidas para controlar custos, cronograma e funcionalidades. A institucionalização dessas práticas é conseguida tanto pela consolidação de práticas existentes na empresa, quanto pela adoção das práticas sugeridas no CMM.

Nesse contexto, o SERPRO definiu procedimentos para todas as áreas-chave do CMM, além de um processo de desenvolvimento único para toda a organização, denominado PSDS, que foi concebido com o objetivo de organizar um conjunto de atividades relacionadas com a criação, certificação, entrega e manutenção de soluções de software. O PSDS define $o$ que fazer (artefatos), quando e como executar atividades de desenvolvimento (fluxos), que ferramentas utilizar (meios), e quem está envolvido no processo (papéis).

Além das atividades típicas de engenharia de software, o PSDS inclui em sua primeira versão todas as áreas-chave ${ }^{2}$ do nível 2 do CMM. A associação entre áreas-chave e atividades constituiu a base para as seguintes melhorias alcançadas no processo: (a) melhoria da qualidade do produto final, (b) controle adequado dos requisitos de software, (c) rastreabilidade dos dados, $(d)$ controle maior dos líderes sobre seus projetos, e $(e)$ um aumento significativo na satisfação do cliente. O grande diferencial do PSDS reside na sua natureza viva, calcada na concepção de um produto de software que, como tal, pode ser mantido e evoluído. Para garantir a atualidade da informação, as tradicionais folhas de papel foram substituídas por páginas Web que compõem um site constantemente atualizado por meio do feedback de seus usuários e de ações desempenhadas por grupos corporativos. Outro ponto de destaque no projeto do PSDS é o conceito de sites de publicação [1], por meio dos quais os produtos (artefatos) gerados ao longo do ciclo de vida de um projeto ficam disponíveis a todos os envolvidos.

\section{Estratégia de Implantação}

Implantar modelos especializados e custosos como o CMM numa empresa da complexidade do SERPRO requer um cuidadoso planejamento de atividades. Estabelecer uma estratégia de trabalho é vital para que o processo de melhoria da qualidade tenha êxito. No

\footnotetext{
${ }^{1}$ O SEI (Software Engineering Institute) foi criado na Carnegie Mellon University em 1984 com o objetivo de apoiar o processo de melhoria de software do Departamento de Defesa Americano.

${ }^{2}$ Gerências de Requisitos, Gerência de Configuração, Gerência de Subcontratação, e Garantia de Qualidade de Software. No PSDS, as demais áreas-chave Planejamento e Acompanhamento de Projeto, originalmente separadas no CMM, foram fundidas numa única área-chave denominada Gerência de Projeto.
} 
caso SERPRO, a estratégia definida foi organizada numa seqüência de etapas, descritas a seguir:

e1. Estruturação. Inicialmente, de acordo com a estrutura organizacional e geográfica do SERPRO, foram definidos grupos de trabalho envolvendo todas as unidades de negócio:

- GEP (Grupo Executor do Projeto) - formado por pessoas-chave de cada uma das unidades de gestão. Traça as diretrizes e aprova as decisões e definições correlatas à implantação do PMoD.

- GTS (Grupo de Trabalho Serpro) - grupos corporativos cuja função é definir a política, os processos e os procedimentos de cada uma das áreas-chave do nível 2 do CMM. Além desses grupos, foi criado um GTS específico para o Processo de Engenharia de Software.

- GTI (Grupo de Trabalho Interno) - projeções dos GTS nas unidades de gestão formadas por profissionais de alto nível, responsáveis pela elaboração e revisão do conteúdo do PSDS.

e2. Definição. Cada GTS definiu políticas organizacionais relativas a sua área-chave. Com base nessas políticas, os GTI levantaram as melhores práticas das suas respectivas unidades de gestão, além de oportunidades de melhoria. Posteriormente, foi feita uma pré-avaliação para verificar a situação atual de cada unidade, e consolidar as práticas e oportunidades identificadas. Após essa fase, o GTS consolidou o material de cada GTI como insumo para a definição do Processo de Software.

e3. Sensibilização. Definido o processo, o foco se transfere para a mobilização do corpo produtivo da empresa. Conforme evidenciado em [5], a tentativa de conduzir um processo bottom-up de melhoria da qualidade leva à formação de ilhas de excelência, ao invés do desejado aumento na capacidade produtiva da organização. Tendo esse princípio como base, um movimento top-down de sensibilização foi executado em toda a empresa, iniciando-se no corpo gerencial, ao que se seguiu o corpo funcional, numa campanha maciça incentivada pelo Presidente do SERPRO, cujo apoio foi primordial para o êxito da implantação.

e4. Institucionalização. Um grupo de Engenharia de Software ficou encarregado de institucionalizar o processo, ou seja, prover treinamento e suporte aos usuários, além de zelar pela sua melhoria contínua. Um grupo de desenvolvedores em projetos-piloto passou a aplicar os padrões e procedimentos definidos, recebendo treinamentos para atuar posteriormente como disseminadores locais. Adicionalmente, o feedback provido por esses profissionais é usado continuamente para a melhoria do processo.

e5. Validação. Numa última fase, grupos de Garantia de Qualidade de Software (GQS) garantem a aplicação do processo, validando os produtos e atividades com base nos padrões definidos. Os GQS também institucionalizam o processo de revisão, servindo de elementos difusores do processo na cultura da organização.

\section{Implementação das Áreas-Chave}

Esta seção descreve a experiência de aplicação da estratégia definida no ambiente do SERPRO. Inicialmente são descritas ferramentas de apoio utilizadas nesse processo, sem as 
quais sua implantação não seria possível. Em seguida, cada uma das áreas-chave é comentada do ponto de vista das dificuldades e soluções encontradas.

\subsection{Ferramental de Apoio}

Evidenciada a necessidade de ferramental para suporte ao processo, o critério adotado foi o de aproveitar as ferramentas já existentes na organização, adquirindo ou construindo aquelas não existentes. A seguir a relação de ferramentas utilizadas:

\begin{tabular}{l|l|l}
\hline Ferramenta(s) & Área-chave & Função \\
\hline SOLICITA & $\begin{array}{l}\text { Gerência de } \\
\text { Projeto }\end{array}$ & $\begin{array}{l}\text { Controle do cadastramento das solicitações pelo usuário. Foi } \\
\text { desenvolvida a partir de uma customização da ferramenta } \\
\text { ClearQuest }\end{array}$ \\
Rational num projeto conjunto entre o SERPRO e a $^{\circledR}$
\end{tabular}

Tabela1. Ferramentas de apoio.

Adicionalmente, o pacote TeamTest ${ }^{\circledR}$ de ferramentas da Rational foi utilizado para aumentar a qualidade dos testes, garantindo a conformidade do projeto com os requisitos especificados. A gerência de qualidade de software foi suportada num primeiro momento pela adoção de artefatos de acompanhamento manual (planilhas, modelos de relatório). Atualmente, encontra-se em fase final de desenvolvimento a ferramenta REVISA que automatiza os processos dessa área-chave.

\subsection{Gerência de Requisitos}

Processos de engenharia de requisitos são dominados por fatores humanos, sociais e organizacionais, envolvendo pessoas com diferentes formações culturais, além de objetivos organizacionais (e individuais) diversos. Os paradigmas individuais geram filtros no processo de comunicação, prejudicando a especificação dos requisitos. Por todos estes aspectos, a Gerência de Requisitos foi a área-chave com o maior grau de dificuldade na implantação do CMM-Nível 2 no SERPRO. Sua institucionalização provocou uma grande mudança cultural na organização, a qual somente pôde ser alcançada mediante $(i)$ a conscientização do corpo funcional do papel fundamental de uma gerência de requisitos; (ii) o treinamento em larga escala nas técnicas e ferramentas de engenharia de requisitos; e finalmente (iii) sua efetiva utilização, cujos benefícios práticos extinguiram os últimos focos de resistência. Dentre estes benefícios, citamos os principais: (a) melhoria da qualidade da especificação; $(b)$ facilidade de documentação e compartilhamento dos requisitos por todos os envolvidos; (c) maior visibilidade por parte de clientes e líderes do que foi solicitado e dos impactos de alteração 
nos requisitos; $(d)$ antecipação de pontos críticos do projeto; $(e)$ resgate ágil do histórico das definições do cliente; e o $(f)$ planejamento antecipado dos testes a partir dos requisitos;

\subsection{Gerência de Projetos}

A falta de uma cultura forte em planejamento e acompanhamento de projeto gerou forte resistência por parte dos líderes de projeto. Muitos líderes temiam que as práticas de gerência de projeto fossem, ao contrário dos seus objetivos, comprometer seus cronogramas de projeto. Essa e outras "má-interpretações" foram contornadas pela inserção do corpo gerencial em workshops de sensibilização e treinamentos em Gerência de Projeto. Ao longo do processo, foi evidenciada a importância de um relacionamento estreito entre representantes dos desenvolvedores e os demais grupos afetados (clientes, fornecedores, unidades de infraestrutura, representantes dos usuários, outros gerentes de projetos de software) para o sucesso da gerência de projeto.

Com a implantação das práticas, uma maior visibilidade do projeto foi logo alcançada tanto pela gerência quanto pelos desenvolvedores, o que pôde ser comprovado na melhoria da qualidade da documentação do projeto, e pela possibilidade de priorização e o tratamento antecipado dos pontos críticos (riscos) do projeto. Um dos fatores que aceleraram a implantação dessa área-chave foi a criação de roteiros e diagramas gráficos mostrando a seqüência de atividades requeridas pelo planejamento e acompanhamento de projeto, e seu interelacionamento com as demais área-chave do CMM.

\subsection{Gerência de Configuração de Software}

A existência de uma cultura prévia de gerenciamento de configuração na empresa facilitou sobremaneira a consolidação desta área-chave, e impulsionou a adoção de suas práticas na totalidade dos projetos. Com isso, houve uma significativa redução na perda de programas e documentos nos ambientes de desenvolvimento e produção. O controle de versões e de baselines permitiu também a visualização do histórico dos projetos, facilitando inclusive as tarefas de gerência do projeto. A definição de uma sistemática para guarda e distribuição de programas, considerando-se as características de cada ferramenta adotada, teve um papel fundamental nesse processo.

\subsection{Garantia de Qualidade de Software}

Esta área-chave é essencial para garantir que os projetos de desenvolvimento sigam as normas e os procedimentos especificados, e que os artefatos produzidos estejam em conformidade com os padrões descritos no PSDS. Os consultores de GQS são os responsáveis por verificar a obediência a estes padrões, e encaminhar as "não-conformidades" levantadas para as lideranças de projeto. O levantamento de inconsistências é realizado por meio de revisões pré-acordadas (ou não) com os líderes de projeto. Um prazo é acordado entre estes e o consultor para a correção das inconsistências. Não-conformidades vigentes após o prazo estabelecido são reportadas à gerência sênior para ciência e providências. Em persistindo a não-conformidade, a ocorrência é reportada diretamente pelo GQS do Pólo ao GQS da UG para acompanhamento e cobrança, sempre sobre a regência de um prazo-limite. Ocorrências em aberto sob jurisdição do GQS da UG e acima do prazo-limite são encaminhadas à última instância da Garantia de Qualidade no SERPRO: o GQS Corporativo, o qual está diretamente vinculado à presidência da empresa. Esse esquema garante a adequação de todos os projetos aos princípios e conceitos estabelecidos no PSDS. As revisões periódicas sobre os projetos 
representam um momento de aprendizado para todos os envolvidos, uma vez que as equipes são orientadas pelos consultores a como melhor utilizar o PSDS.

\subsection{Gerência de Subcontratação}

Os processos, métodos e procedimentos encontram-se definidos para esta área-chave. Contudo, não tivemos oportunidade de exercer a gerência de subcontratação de software, e coletar os resultados de sua aplicação.

\section{Lições Aprendidas}

Implantar o nível 2 do CMM numa empresa como o SERPRO não é uma tarefa fácil, mas os esforços aqui descritos para sua consecução provam que ela pode ser atingida com sucesso. Certamente essa é uma experiência muito enriquecedora para todos os envolvidos, a qual traz lições que podem ser assimiladas e (na medida do possível) repetidas em outros ambientes. A seguir, destacamos as principais lições aprendidas com o processo SERPRO:

a. A infra-estrutura de hardware e software requerida para o suporte à implantação deve ser considerada o mais cedo possível no processo, devido ao elevado custo de sua aquisição e instalação. É importante investir no aproveitamento das ferramentas já existentes na organização;

b. Equipes devem ser formadas para dar suporte operacional ao novo ambiente e às ferramentas adotadas. O bom aproveitamento do ambiente depende fundamentalmente do desempenho destes profissionais;

c. É imprescindível a alocação de recursos para formação de grupos de engenharia de software (SEPG) e de garantia de qualidade (GQS), a despeito da pressão pelo atendimento a demandas ou da impossibilidade de contratação de novos recursos. A contribuição desses grupos para o processo como um todo compensa o esforço de alocação da mão-de-obra;

d. O investimento em larga escala para treinamento dos desenvolvedores é mais que uma necessidade. É essencial;

e. A revisão periódica do trabalho é encarada muitas vezes como uma invasão dos direitos individuais pelos desenvolvedores, os quais não estão acostumados a terem seu trabalho questionado por uma pessoa externa ao projeto. Assim, é extremamente importante que o gerente de qualidade seja uma pessoa tecnicamente preparada, com habilidades para estabelecer um bom relacionamento interpessoal;

f. Nunca implantar cada área-chave separadamente, devido à forte dependência entre elas;

g. A tendência a ir diretamente para a fase de projeto deve ser combatida nos desenvolvedores por meio do fortalecimento dos processos de requisitos. Uma vez aplicados de maneira correta, os benefícios práticos sentidos no projeto fazem o restante do trabalho;

h. É importante o planejamento e a participação das lideranças, e fundamental o apoio da alta administração. Juntos eles garantem o rumo do projeto na direção certa;

i. Meios de documentação dos resultados como os sites de publicação simplificam enormemente o trabalho dos gerentes de qualidade, liberando os líderes para as tarefas específicas de gerência de projeto. Posteriormente, uma reunião é o suficiente para a discussão entre as partes; 
j. Instrutorias Internas proporcionam treinamentos mais voltados para a realidade da organização, incorporando exemplos práticos que facilitam a assimilação dos princípios técnicos e gerenciais. A redução dos custos com treinamento é outro fator atrativo desse tipo de prática;

k. Nunca perder de vista uma das peças fundamentais no processo de melhoria: o cliente. Os clientes são um dos afetados pelas (e mais interessados nas) mudanças geradas, portanto o envolvimento e aceitação do processo por parte do cliente é um passaporte para a internalização do processo entre as equipes;

1. Implantar as práticas-chave em projetos recém iniciados provoca um aumento imediato da satisfação do cliente. Porém, a sua extensão aos sistemas legados, cuja manutenção e processo evolutivo continuam vivos, é fundamental para consolidar a melhoria do processo de software em toda a empresa;

m. Os profissionais integrados a instituições acadêmicas por meio de cursos de Especialização e Pós-Graduação têm muito a contribuir com o processo, em especial se estes cursos são focados na qualidade de software. Essa prática deve ser incentivada em todas as empresas.

\section{Conclusão}

A implantação de processos de melhoria de qualidade é mais que uma tendência nas empresas de software, é um requisito primordial para enfrentar o mercado competitivo e atender às exigências de seus clientes. A experiência do SERPRO na implantação do nível 2 do CMM demonstra que, apesar das dificuldades inerentes a este tipo de processo, e da complexidade adicional de sua estrutura organizacional, é possível implantar com sucesso todas as suas práticas. O planejamento bem estruturado desde o início está por trás desse sucesso.

Ao longo de 2 anos de trabalho, uma das mais importantes lições aprendidas é que quando o processo trabalha para as pessoas, as pessoas trabalham para o processo. A mobilização de todos os envolvidos é crucial para garantir uma melhoria homogênea do processo de desenvolvimento, e somente pode ser conseguida quando cada profissional percebe benefícios na sua utilização.

\section{Referências}

[1] Paim, F. R. S., Tavares, H. C. "Implementing a Living Software Process", in Proceedings of the ICSE'2002 Workshop on Software Quality (WoSQ), Maio, 2002.

[2] Paulk, M. C. et al. "Key Practices of the Capability Maturity Model", Version 1.1, Technical Report SEI-CMU-93-TR-25, Software Engineering Institute, 1993.

[3] Paulk, M. C. et al. "The Capability Maturity Model: Guidelines for improving the Software Process", Carnegie Mellon University, Software Engineering Institute, ISBN 0201-54664-7, Addison-Wesley, MA, 1995.

[4] Linger, R. C. et al. "Cleanroom Software Engineering: implementation of the Capability Maturity Model for Software", Technical Report SEI-CMU-96-TR-23. Software Engineering Institute, 1996.

[5] Paulk, M. C. "Effective CMM-Based Software Improvement", in Proceedings of the $6^{\text {th }}$ International Conference on Software Quality, Ottawa, Canadá, Outubro, 1996, pp. 226237. 